PROCEEDINGS OF THE

AMERICAN MATHEMATICAL SOCIETY

Volume 132, Number 9, Pages 2567-2575

S 0002-9939(04)07271-5

Article electronically published on April 8, 2004

\title{
THE S-ELEMENTARY FRAME WAVELETS ARE PATH CONNECTED
}

\author{
X. DAI, Y. DIAO, Q. GU, AND D. HAN
}

(Communicated by David R. Larson)

\begin{abstract}
An s-elementary frame wavelet is a function $\psi \in L^{2}(\mathbb{R})$ which is a frame wavelet and is defined by a Lebesgue measurable set $E \subset \mathbb{R}$ such that $\hat{\psi}=\frac{1}{\sqrt{2 \pi}} \chi_{E}$. In this paper we prove that the family of s-elementary frame wavelets is a path-connected set in the $L^{2}(\mathbb{R})$-norm. This result also holds for s-elementary $A$-dilation frame wavelets in $L^{2}\left(\mathbb{R}^{d}\right)$ in general. On the other hand, we prove that the path-connectedness of s-elementary frame wavelets cannot be strengthened to uniform path-connectedness. In fact, the sets of normalized tight frame wavelets and frame wavelets are not uniformly path-connected either.
\end{abstract}

\section{INTRODUCTION}

Let $L^{2}(\mathbb{R})$ be the set of Lebesgue square integrable functions on $\mathbb{R}$. The Fourier transform for $f \in L^{2}(\mathbb{R}) \cap L^{1}(\mathbb{R})$ is defined by

$$
\frac{1}{\sqrt{2 \pi}} \int_{\mathbb{R}} e^{-i s t} f(t) d t
$$

This is denoted by $\mathcal{F} f$ or $\widehat{f}$. It is known that $\mathcal{F}$ can be uniquely extended to a unitary operator on $L^{2}(\mathbb{R})$. Let $D$ and $T$ be the dilation and translation operators on $L^{2}(\mathbb{R})$, namely $(D f)(x)=\sqrt{2} f(2 x)$ and $(T f)(x)=f(x-1)$ for any $f \in L^{2}(\mathbb{R})$. We will use $\widehat{D}, \widehat{T}$ for the product $\mathcal{F} D \mathcal{F}^{-1}$ and $\mathcal{F} T \mathcal{F}^{-1}$. It is known that $\widehat{D}=D^{-1}$ and $\widehat{T} f(t)=e^{-i s t} f(t)\left[3\right.$. A function $\psi \in L^{2}(\mathbb{R})$ is called a frame wavelet for $L^{2}(\mathbb{R})$ if there exist two positive constants $0<a \leq b$ such that for any $f \in L^{2}(\mathbb{R})$,

$$
a\|f\|^{2} \leq \sum_{n, \ell \in \mathbb{Z}}\left|\left\langle f, D^{n} T^{\ell} \psi\right\rangle\right|^{2} \leq b\|f\|^{2} .
$$

If one can choose $a=b$ in (1), then $\psi$ is called a tight frame wavelet. Furthermore, if $a=b=1$, then $\psi$ is called a normalized tight frame wavelet. Let $E$ be a Lebesgue measurable set of finite measure, and $\chi_{E}$ the corresponding characteristic function. If the function $\psi_{E} \in L^{2}(\mathbb{R})$ defined by $\widehat{\psi_{E}}=\frac{1}{\sqrt{2 \pi}} \chi_{E}$ is a frame wavelet, a tight frame wavelet or a normalized tight frame wavelet for $L^{2}(\mathbb{R})$, then the set $E$ is called a frame wavelet set, a tight frame wavelet set or a normalized tight frame wavelet set

Received by the editors March 8, 2002 and, in revised form, February 20, 2003.

2000 Mathematics Subject Classification. Primary 46N99.

Key words and phrases. Frames, wavelets, frame wavelets, frame wavelet sets, Fourier transform. 
for $L^{2}(\mathbb{R})$, respectively. The corresponding function $\psi_{E}$ is called an s-elementary, a tight s-elementary or a normalized tight s-elementary frame wavelet. The name s-elementary is borrowed from [3], [8], where a wavelet whose Fourier transform is of the form $\frac{1}{\sqrt{2 \pi}} \chi_{E}$ is called an s-elementary wavelet.

The topological property of various families of wavelets is an interesting topic in the study of wavelet theory. In [3], the question concerning the path-connectedness of the set of all orthonormal wavelets was raised. Similar questions were raised and studied in [7, [9] about the set of all MRA-wavelets, tight frame wavelets and MRA tight frame wavelets. In fact, discussions on such issues can be traced a few years back before the publication of [3]. One can ask similar questions on the families of normalized tight frame wavelets and frame wavelets. These turn out to be very hard questions, and all of them remain unsolved at this time. However, it has been proved that the family of s-elementary (orthonormal) wavelets is pathconnected in 8], and it has since been shown that the set of all MRA-wavelets is also path-connected [6], 9]. In [2], the authors proved that the set of normalized tight s-elementary frame wavelets is path-connected.

In this paper, we are mainly concerned with the path-connectedness of the set of s-elementary frame wavelets. Showing the path-connectedness of the set of selementary frame wavelets can potentially lend a helping hand in proving the pathconnectivity of the set of all frame wavelets, since one would only need to show that any frame wavelet is path-connected to an s-elementary frame wavelet. Since the set of normalized tight s-elementary frame wavelets is path-connected, it seems plausible that the set of s-elementary frame wavelets may also be path-connected. However, proving it is not trivial. The reason is that the proof in 2] relies on the characterization of the normalized tight frame wavelet sets (which is given in [1]). Yet, the characterization of frame wavelet sets is still an open question at this time. So, it is somewhat surprising that we are able to use the partial results about frame wavelet sets developed in [1] to prove that the set of s-elementary frame wavelets is indeed path-connected. This result can be generalized (by using a similar argument and some results from [2]) to s-elementary frame wavelets in higher dimensional cases with arbitrary expansive matrix dilations. This is done in Section 3. In the last section, we discuss the uniform path-connectivity of the sets of frame wavelets, normalized tight frame wavelets and s-elementary frame wavelets. We prove that none of these sets is uniformly path-connected.

We need to point out that our result only applies to the s-elementary frame wavelets so defined here. Since the result of [8] can easily be extended to the set of all MSF-wavelets, it is natural to ask whether our method can be extended to show the path-connectedness of the set of all frame wavelets whose Fourier transforms are supported on frame sets (the term MSF no longer applies in the frame wavelet case, since the support of a frequency frame wavelet can be as small as possible). At this time, we are unable to do this. Again, the major obstacle is the lack of a characterization of frequency frame wavelets.

\section{BASIC CONCEPTS AND LEMMAS}

Throughout this paper, we only deal with subsets of $\mathbb{R}$ that are Lebesgue measurable. Thus, in all lemmas and theorems, it is understood that all sets involved are Lebesgue measurable. Most definitions and the proofs of the lemmas in this section can be found in [1] and [2]. Please refer to these two papers for the details. 
Let $E$ be a Lebesgue measurable set in $\mathbb{R}$. A point $x \in E$ is said to have a dilation index $\delta_{E}(x)=k$ if there are exactly $k$ points in the set $E \cap\left(\bigcup_{n \in \mathbb{Z}} 2^{n} x\right)$. For each fixed natural number $k$, the set $E(\delta, k)=\left\{x \in E: \delta_{E}(x)=k\right\}$ is Lebesgue measurable. Furthermore, each $E(\delta, k)$ is a disjoint union of $k$ measurable sets $\left\{E^{j}(\delta, k): 1 \leq j \leq k\right\}$ such that $\delta_{E^{j}(\delta, k)}(x)=1$ for each point $x \in E^{j}(\delta, k)$. Similarly, a point $x \in E$ is said to have a $2 \pi$-translation index $\tau_{E}(x)=k$ if there are exactly $k$ points in the set $E \cap\left(\bigcup_{n \in \mathbb{Z}}(2 \pi n+x)\right)$. For each fixed natural number $k$, the set $E(\tau, k)=\left\{x \in E: \tau_{E}(x)=k\right\}$ is Lebesgue measurable and is a disjoint union of $k$ measurable sets $\left\{E^{j}(\tau, k): 1 \leq j \leq k\right\}$ such that $\tau_{E^{j}(\tau, k)}(x)=1$ for each point $x \in E^{j}(\tau, k)$. If there is a number $M$ such that $E(\delta, k)$ and $E(\tau, k)$ are null sets for all $k>M$, the set $E$ is called a basic set.

Assume further that $E$ is of finite measure. For any $f \in L^{2}(\mathbb{R})$, define

$$
\left(H_{E} f\right)(s)=\sum_{n, \ell \in \mathbb{Z}}\left\langle f, \widehat{D}^{n} \widehat{T}^{\ell} \frac{1}{\sqrt{2 \pi}} \chi_{E}\right\rangle \widehat{D}^{n} \widehat{T}^{\ell} \frac{1}{\sqrt{2 \pi}} \chi_{E}(s) .
$$

A set $E$ is called a Bessel set if $H_{E} f$ converges in norm unconditionally for each $f \in L^{2}(\mathbb{R})$ and $\left\langle H_{E} f, f\right\rangle \leq B\|f\|^{2}$ for some constant $B>0$. Theorem 1 of [1] implies the following lemma.

Lemma 1. A set $E$ is Bessel if and only if it is a basic set. Moreover, if $\mu(E(\delta, m))$ $=\mu(E(\tau, m))=0$ for all $m>M$ (where $\mu$ is the Lebesgue measure), then $\left\langle H_{E} f, f\right\rangle$ $\leq M^{5 / 2}\|f\|^{2}$ for any $f \in L^{2}(\mathbb{R})$.

On the other hand, the same argument used in the proof of Theorem 2 in [1] leads us to the following lemma.

Lemma 2. Let $E$ be a basic set. Assume that $\Omega=\bigcup_{k \in \mathbb{Z}} 2^{k} E(\tau, 1)=\bigcup_{k \in \mathbb{Z}} 2^{k} E$. Then

$$
\left\langle H_{E} f, f\right\rangle \geq\|f\|^{2}, \quad \forall f \in L^{2}(\mathbb{R}), \operatorname{supp}(f) \subset \Omega .
$$

Lemma 3 below is obtained by using Lemma 1 and Lemma 2 .

Lemma 3. Let $E$ be a basic set and $E(\tau, m)=E(\delta, m)=\emptyset, \forall m>M$. Let $F$ be a measurable set such that $E \subset \bigcup_{k \in \mathbb{Z}} 2^{k} F$ and $F=F(\tau, 1)$. Then

$$
\left\langle H_{E} f, f\right\rangle \leq M^{5 / 2}\left\langle H_{F} f, f\right\rangle, \quad \forall f \in L^{2}(\mathbb{R}) .
$$

Proof. Define $\Omega=\bigcup_{k \in \mathbb{Z}} 2^{k} F$ and $\Omega_{1}=\mathbb{R} \backslash \Omega$. Let $f \in L^{2}(\mathbb{R})$. Define $f_{1}=f \chi_{\Omega}$ and $f_{2}=f \chi_{\Omega_{1}}$. Then we have $H_{E} f_{2}=H_{F} f_{2}=0$ and $\left\langle H_{E} f_{1}, f_{2}\right\rangle=\left\langle H_{F} f_{1}, f_{2}\right\rangle=$ 0 . Hence $\left\langle H_{E} f, f\right\rangle=\left\langle H_{E} f_{1}, f_{1}\right\rangle \leq M^{5 / 2}\left\|f_{1}\right\|^{2}$ by Lemma 1. and $\left\langle H_{F} f, f\right\rangle=$ $\left\langle H_{F} f_{1}, f_{1}\right\rangle \geq\left\|f_{1}\right\|^{2}$ by Lemma 2. The result follows.

For any $E \subset \mathbb{R}$, let $\tau(E)=\bigcup_{\mathbb{Z}}(E+2 k \pi)$. Be careful not to confuse $\tau(E)$ with $\tau_{E}(x)$, the translation index of $x$ in $E$. We say that two sets $E$ and $F$ are $2 \pi$ translation disjoint if $\tau(E) \cap \tau(F)=\emptyset$. The following lemma is obtained from Lemma 5 of [1].

Lemma 4. If $E$ and $F$ are $2 \pi$-translation disjoint basic sets, then

$$
H_{E \cup F} f=H_{E} f+H_{F} f, \quad \forall f \in L^{2}(\mathbb{R}) .
$$

It is well-known that if $\psi=\psi_{E}$, then (11) is equivalent to

$$
a\|f\|^{2} \leq \sum_{n, \ell \in \mathbb{Z}}\left|\left\langle f, \widehat{D}^{n} \widehat{T}^{\ell} \frac{1}{\sqrt{2 \pi}} \chi_{E}\right\rangle\right|^{2} \leq b\|f\|^{2} .
$$


Combining this with (2), we get

Lemma 5. For $\psi=\psi_{E}$, (1) is equivalent to

$$
a\|f\|^{2} \leq\left\langle H_{E} f, f\right\rangle \leq b\|f\|^{2}, \quad \forall f \in L^{2}(\mathbb{R}) .
$$

Finally, we will need the following lemma in proving our main theorem in the next section. This lemma is the one-dimensional case of Theorem 4 in [2].

Lemma 6. The family of normalized tight s-elementary frame wavelets is pathconnected under the $L^{2}(\mathbb{R})$ norm.

\section{THE MAIN THEOREM AND ITS PROOF}

In this section we prove our main result.

Theorem 1. The family of s-elementary frame wavelets is path-connected under the $L^{2}(\mathbb{R})$ norm.

Proof. We will prove that for a given frame wavelet set $E$, there is a continuous path of the form $\chi_{W_{t}}$ connecting $\chi_{E}$ to $\chi_{F}$, where each $W_{t}$ is a frame wavelet set and $F$ is a normalized tight frame set. This implies that each s-elementary frame wavelet is connected by a continuous path (of s-elementary frame wavelets) to a normalized tight s-elementary frame wavelet. This in turn implies the theorem, by Lemma 6 .

Let $E$ be a frame wavelet set and $\psi_{E}$ the corresponding s-elementary frame wavelet. $E$ is a Bessel set, hence a basic set by Lemma 1. So there is a number $M$ such that $E(\tau, m)=E(\delta, m)=\emptyset, \forall m>M$. Thus we can choose $B=M^{5 / 2}$ in (11) by Lemma 1 Let $a>0$ be the lower frame bound of $\psi_{E}$. We have $a\|f\|^{2} \leq$ $\left\langle H_{E} f, f\right\rangle \leq M^{5 / 2}\|f\|^{2}$ for all $f \in L^{2}(\mathbb{R})$ by Lemma 5 . Let $m_{0}$ be a positive integer large enough so that $M / 2^{m_{0}}<1 / 4$. Let

$$
F=\left[-\frac{2 \pi}{2^{m_{0}+1}},-\frac{\pi}{2^{m_{0}+1}}\right) \cup\left[\frac{\pi}{2^{m_{0}+1}}, \frac{2 \pi}{2^{m_{0}+1}}\right) .
$$

By Corollary 3 of [1], the set $F$ is a normalized tight frame set. It is left to the reader to verify that $E \cup F$ is a basic set and every measurable subset of $E \cup F$ is a basic set.

For any $s \in E$, there is a unique integer $k(s)$ such that $s / 2^{k(s)} \in F$. Thus $h(s)=s / 2^{k(s)}$ defines a mapping from $E$ to $F$. We leave it to our reader to prove that the image of each measurable subset in $E$ under $h$ is measurable. Furthermore, if $E^{\prime}$ is a subset of $E \cap \mathbb{R} \backslash[-\pi, \pi]$, then $\mu\left(h\left(E^{\prime}\right)\right)<\frac{1}{2^{m_{0}+1}} \mu\left(E^{\prime}\right)$. Define

$$
\begin{aligned}
& F_{t}^{0}=\left[-\frac{2 \pi}{2^{m_{0}+1}},-\frac{(2-t) \pi}{2^{m_{0}+1}}\right] \cup\left[\frac{\pi}{2^{m_{0}+1}}, \frac{(1+t) \pi}{2^{m_{0}+1}}\right], \\
& F_{t}^{1}=h\left(\tau\left(F_{t}^{0}\right) \cap\left(E \backslash F_{t}^{0}\right)\right), \\
& F_{t}^{2}=h\left(\tau\left(F_{t}^{1}\right) \cap\left(E \backslash F_{t}^{1}\right)\right), \\
& \cdots \\
& F_{t}^{n}=h\left(\tau\left(F_{t}^{n-1}\right) \cap\left(E \backslash F_{t}^{n-1}\right)\right), \\
& \cdots \\
& F_{t}=\bigcup_{k \geq 0} F_{t}^{k}, t \in[0,1] .
\end{aligned}
$$


Notice that the set $F_{t}$ is a measurable subset of $F$; hence it is a basic set. Let $E_{t}=\tau\left(F_{t}\right) \cap E$. It is clear that any point in $\tau\left(E_{t}\right)$ must be in $\tau\left(F_{t}\right)$, hence cannot be in $\tau\left(E \backslash E_{t}\right)$. So the sets $E_{t}$ and $E \backslash E_{t}$ are $2 \pi$-translation disjoint. By Lemma 4 we have

$$
H_{E} f=H_{E_{t}} f+H_{E \backslash E_{t}} f .
$$

Hence

$$
\left\langle H_{E} f, f\right\rangle=\left\langle H_{E_{t}} f, f\right\rangle+\left\langle H_{E \backslash E_{t}} f, f\right\rangle \geq a\|f\|^{2} .
$$

Similarly,

$$
H_{F_{t} \cup\left(E \backslash E_{t}\right)} f=H_{F_{t}} f+H_{E \backslash E_{t}} f,
$$

since $F_{t}$ and $E \backslash E_{t}$ are also $2 \pi$-translation disjoint. It follows that

$$
\left\langle H_{F_{t} \cup\left(E \backslash E_{t}\right)} f, f\right\rangle=\left\langle H_{F_{t}} f, f\right\rangle+\left\langle H_{E \backslash E_{t}} f, f\right\rangle .
$$

Notice that $F_{t}=F_{t}(\tau, 1)$, since $F_{t} \subset F$ and $F=F(\tau, 1)$. Let $x \in E_{t}=E \cap \tau\left(F_{t}\right)$. If $x \notin F_{t}$, then $x \in \tau\left(F_{t}^{n}\right) \cup\left(E \backslash F_{t}^{n}\right)$ for some $n \geq 0$. So $h(x) \in F_{t}^{n+1} \subset F_{t}$. Hence we have

$$
E_{t} \subset \bigcup_{k \in \mathbb{Z}} 2^{k} F_{t}
$$

By Lemma 3 we have

$$
\left\langle H_{F_{t}} f, f\right\rangle \geq M^{-\frac{5}{2}}\left\langle H_{E_{t}} f, f\right\rangle .
$$

Now define $W_{t}=F_{t} \cup\left(E \backslash E_{t}\right)$. Since $W_{t} \subset F \cup E$, it is a basic set. By Lemma 1 there is a positive number $B$ (independent of $t$ ) such that

$$
\left\langle H_{W_{t}} f, f\right\rangle \leq B\|f\|^{2}, \quad \forall f \in L^{2}(\mathbb{R}) .
$$

On the other hand, (5), (6) and (8) imply that

$$
\begin{aligned}
\left\langle H_{W_{t}} f, f\right\rangle & =\left\langle H_{F_{t}} f, f\right\rangle+\left\langle H_{E \backslash E_{t}} f, f\right\rangle \\
& \geq M^{-\frac{5}{2}}\left\langle H_{E_{t}} f, f\right\rangle+\left\langle H_{E \backslash E_{t}} f, f\right\rangle \\
& >M^{-\frac{5}{2}}\left(\left\langle H_{E_{t}} f, f\right\rangle+\left\langle H_{E \backslash E_{t}} f, f\right\rangle\right) \\
& \geq a M^{-\frac{5}{2}}\|f\|^{2} .
\end{aligned}
$$

Therefore, $W_{t}$ is a frame wavelet set for each $t \in[0,1]$. It is easy to verify that $W_{0}=E$ (since $F_{0}=E_{0}=\emptyset$ ) and $W_{1}=F \cup(E \backslash \tau(F)$ ). Notice that $F, E \backslash \tau(F)$ are $2 \pi$-translation disjoint. Thus, by Lemma 2 , for any measurable subset $G$ of $E \backslash \tau(F), F \cup G$ is a frame set since $\bigcup_{k \in \mathbb{Z}} 2^{k} F=\mathbb{R}$. In particular, if we let $G_{t}=$ $\left(-\tan \left(\frac{\pi}{2} t\right), \tan \left(\frac{\pi}{2} t\right)\right) \cap(E \backslash \tau(F))$, then $F \cup G_{t}$ is a frame set. We leave it to our reader to verify that the mapping $t \longrightarrow \chi_{F \cup G_{t}}$ is continuous in norm. Since $G_{0}=\emptyset$ and $G_{1}=E \backslash \tau(F)$, this defines a continuous path from $\chi_{F}$ to $\chi_{W_{1}}$. Therefore, to complete the proof of Theorem 1, it suffices to show that the mapping $t \longrightarrow \chi_{W_{t}}$ is continuous in norm. We will achieve this in a few steps.

Step 1: We first show that the mapping $t \rightarrow \chi_{F_{t}}$ is continuous in norm. For $0 \leq t \leq 1$, we have $\mu\left(F_{t}^{0}\right) \leq \pi / 2^{m_{0}}$. By the property of $E$, for a point $s \in F_{t}^{0}$, the set $\{s+2 k \pi: k \in \mathbb{Z}\} \cap E$ has at most $M$ points. This implies that

$$
\mu\left(\tau\left(F_{t}^{0}\right) \cap\left(E \backslash F_{t}^{0}\right)\right) \leq M \mu\left(F_{t}^{0}\right) .
$$


Since $\tau\left(F_{t}^{0}\right) \cap\left(E \backslash F_{t}^{0}\right) \subset \mathbb{R} \backslash[-\pi, \pi]$, it follows from (10) that

$$
\begin{aligned}
\mu\left(F_{t}^{1}\right) & \leq \frac{1}{2^{m_{0}}+1} \mu\left(\tau\left(F_{t}^{0}\right) \cap\left(E \backslash F_{t}^{0}\right)\right) \\
& \leq \frac{M}{2^{m_{0}+1}} \mu\left(F_{t}^{0}\right) \leq \frac{1}{4} \mu\left(F_{t}^{0}\right) .
\end{aligned}
$$

By induction, we have

$$
\mu\left(F_{t}^{n}\right) \leq \frac{M}{2^{m_{0}+1}} \mu\left(F_{t}^{n-1}\right) \leq \frac{1}{4^{n}} \mu\left(F_{t}^{0}\right) .
$$

Therefore, the convergence of $\chi_{\cup_{0 \leq k \leq n} F_{t}^{k}}$ to $\chi_{F_{t}}$ is uniform with respect to $t \in[0,1]$. For any $\epsilon>0$, choose $N>0$ large enough so that $\pi / 4^{N}<\epsilon / 4$; then for any $t \in[0,1]$, we have

$$
\left|\chi_{\cup_{0 \leq k \leq N} F_{t}^{k}}-\chi_{F_{t}}\right| \leq \sum_{k>N} \frac{1}{4^{k}} \mu\left(F_{t}^{0}\right) \leq \frac{\mu\left(F_{t}^{0}\right)}{4^{N}}<\frac{\pi}{4^{N}}<\frac{\epsilon}{4},
$$

since $\mu\left(F_{t}^{0}\right) \leq \pi$ for any $t$. If the mapping $t \rightarrow \chi_{F_{t}^{n}}$ is continuous in norm for each $n$, then $\chi_{\cup_{0 \leq k \leq N} F_{t}^{k}}$ is uniformly continuous on $[0,1]$. Thus, there exists $\delta(\epsilon)>0$ such that $\left|\chi_{\cup_{0 \leq k \leq N}} F_{t_{2}}^{k}-\chi_{\cup_{0 \leq k \leq N} F_{t_{1}}^{k}}\right|<\epsilon / 2$ whenever $\left|t_{2}-t_{1}\right|<\delta(\epsilon)$. It follows that

$$
\begin{aligned}
\mid \chi_{F_{t_{2}}} & -\chi_{F_{t_{1}}}|\leq| \chi_{\cup_{0 \leq k \leq N} F_{t_{2}}^{k}}-\chi_{\cup_{0 \leq k \leq N} F_{t_{1}}^{k}} \mid \\
& +\left|\chi_{\cup_{0 \leq k \leq N} F_{t_{2}}^{k}}-\chi_{F_{t_{2}}}\right|+\left|\chi_{\cup_{0 \leq k \leq N} F_{t_{1}}^{k}}-\chi_{F_{t_{1}}}\right| \\
& \leq \frac{\epsilon}{2}+\frac{\epsilon}{4}+\frac{\epsilon}{4}=\epsilon .
\end{aligned}
$$

That is, $\chi_{F_{t}}$ is also uniformly continuous on $[0,1]$. Therefore, it suffices for us to prove that the mapping $t \rightarrow \chi_{F_{t}^{n}}$ is continuous in norm for each $n$. We will prove this by induction. Clearly, the mapping $t \rightarrow \chi_{F_{t}^{0}}$ is continuous. Assume that it is true for $n$. We will show that it is true for $n+1$. For this purpose, we write $K \Delta L=(K \backslash L) \cup(L \backslash K)$ for any sets $K$ and $L$, and let $D_{t}^{n}=\tau\left(F_{t}^{n}\right) \cap\left(E \backslash F_{t}^{n}\right)$. For any $t, t^{\prime} \in[0,1]$, we claim that $D_{t}^{n} \Delta D_{t^{\prime}}^{n} \subset \tau\left(F_{t}^{n} \Delta F_{t^{\prime}}^{n}\right) \cap E$. Let $s \in D_{t}^{n} \Delta D_{t^{\prime}}^{n}$. We can assume that $s \in D_{t}^{n} \backslash D_{t^{\prime}}^{n}$. Then there is an integer $k$ such that $s+2 k \pi \in F_{t}^{n}$. However, $s \notin F_{t}^{n}$. It follows that $k \neq 0$. Thus $s \notin F_{t^{\prime}}^{n}$, for otherwise we would have both $s$ and $s+2 k \pi \in F_{t^{\prime}}^{n} \cup F_{t}^{n} \subset F \subset[-\pi, \pi)$, which is impossible since $k \neq 0$. Therefore $s \in E \backslash F_{t^{\prime}}^{n}$. Since $s \notin D_{t^{\prime}}^{n}=\tau\left(F_{t^{\prime}}^{n}\right) \cap\left(E \backslash F_{t^{\prime}}^{n}\right)$, it follows that $s \notin \tau\left(F_{t^{\prime}}^{n}\right)$. Hence $s+2 k \pi \in F_{t}^{n} \Delta F_{t^{\prime}}^{n}$, and therefore $s \in \tau\left(F_{t}^{n} \Delta F_{t^{\prime}}^{n}\right) \cap E$, as expected.

We now have

$$
F_{t}^{n+1} \Delta F_{t^{\prime}}^{n+1} \subset h\left(D_{t}^{n} \Delta D_{t^{\prime}}^{n}\right) \subset h\left(\tau\left(F_{t}^{n} \Delta F_{t^{\prime}}^{n}\right) \cap E\right) .
$$

Therefore,

$$
\begin{aligned}
\mu\left(F_{t}^{n+1} \Delta F_{t^{\prime}}^{n+1}\right) & \leq \mu\left(h\left(\left(F_{t}^{n} \Delta F_{t^{\prime}}^{n}\right)^{+} \cap E\right)\right) \\
& \leq \frac{M}{2^{m_{0}+1}} \mu\left(F_{t}^{n} \Delta F_{t^{\prime}}^{n}\right) .
\end{aligned}
$$

(13) implies that the mapping $t \rightarrow \chi_{F_{t}^{n+1}}$ is continuous, since the mapping $t \rightarrow \chi_{F_{t}^{n}}$ is. This completes the proof that the mapping $t \rightarrow \chi_{F_{t}^{n}}$ is continuous in norm for all $n$. Hence the mapping $t \rightarrow \chi_{F_{t}}$ is continuous, as claimed.

Step 2: We now show that the mapping $t \rightarrow \chi_{E_{t}}$ is also continuous. In fact, this follows from the inclusion $E_{t} \Delta E_{t^{\prime}} \subset \tau\left(F_{t} \Delta F_{t^{\prime}}\right) \cap E$, which implies that

$$
\mu\left(E_{t} \Delta E_{t^{\prime}}\right) \leq \mu\left(\tau\left(F_{t} \Delta F_{t^{\prime}}\right) \cap E\right) \leq M \mu\left(F_{t} \Delta F_{t^{\prime}}\right) .
$$


Step 3: Finally, the continuity of $t \rightarrow \chi_{W_{t}}$ follows from the continuity of the mappings $t \rightarrow \chi_{F_{t}}$ and $t \rightarrow \chi_{E \backslash E_{t}}$ and the fact that $F_{t} \cap\left(E \backslash E_{t}\right)=\emptyset$. This completes our proof of Theorem 1 .

In [1], it is shown that the frame bound of an s-elementary tight frame wavelet is a positive integer. If we use $S_{f}$ to denote the set of all s-elementary frame wavelets, $S_{f}(j)$ to denote the set of all s-elementary tight frame wavelets of frame bound $j \geq 1$ (so $S_{f}(1)$ is the set of all s-elementary normalized tight frame wavelets), then it is not hard to see that each $S_{f}(j)$ is path-disconnected from $\bigcup_{k \neq j} S_{f}(k)$. For $j \neq 1$, it remains unclear whether $S_{f}(j)$ is path-connected. This situation is illustrated in Figure 1.

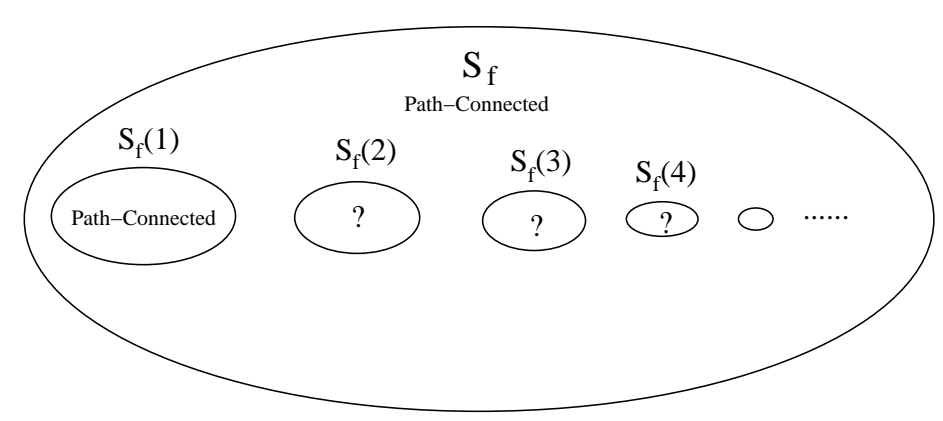

FiguRE 1. Illustration of the path-connectedness of s-elementary frame wavelets.

We now point out that Theorem 1 is also valid for higher dimensional cases. Let $A$ be a $d \times d$ expansive matrix, that is, all eigenvalues of $A$ have norm greater than 1. Let $D_{A}$ be the unitary operator defined by $D_{A} f(s)=|\operatorname{det} A|^{\frac{1}{2}} f(A s)$ and $T_{\ell}$ the unitary operator defined by $T_{\ell} f(s)=f(s-\ell)$, where $f \in L^{2}\left(\mathbb{R}^{d}\right)$ and $\ell \in \mathbb{Z}^{d}$. A function $\psi \in L^{2}\left(\mathbb{R}^{d}\right)$ is called an A-dilation frame wavelet for $L^{2}\left(\mathbb{R}^{d}\right)$ if there exist two positive constants $0<a \leq b$ such that for any $f \in L^{2}\left(\mathbb{R}^{d}\right)$,

$$
a\|f\|^{2} \leq \sum_{n \in \mathbb{Z}, \ell \in \mathbb{Z}^{d}}\left|\left\langle f, D_{A}^{n} T^{\ell} \psi\right\rangle\right|^{2} \leq b\|f\|^{2} .
$$

The Fourier-Plancherel transform $\mathcal{F}$ on $L^{2}\left(\mathbb{R}^{d}\right)$ is a unitary operator such that for $f \in L^{2}\left(\mathbb{R}^{d}\right) \cap L^{1}\left(\mathbb{R}^{d}\right)$,

$$
(\mathcal{F} f)(s)=\frac{1}{(2 \pi)^{d / 2}} \int_{\mathbb{R}^{d}} e^{-i(s \circ t)} f(t) d m
$$

where $s \circ t$ denotes the real inner product. If the function $\psi_{E} \in L^{2}\left(\mathbb{R}^{d}\right)$ defined by $\widehat{\psi}=\frac{1}{(2 \pi)^{d / 2}} \chi_{E}$ for some measurable set $E$ in $\mathbb{R}^{d}$ is a frame wavelet for $L^{2}\left(\mathbb{R}^{d}\right)$, then the function $\psi_{E}$ is called an s-elementary A-dilation frame wavelet. We have

Theorem 2. The family of s-elementary A-dilation frame wavelets is pathconnected in the $L^{2}\left(\mathbb{R}^{d}\right)$ norm. 


\section{LOCAL COMMUTANT AND UNIFORM CONNECTIVITY}

Let $\psi$ be a fixed orthonormal wavelet. The local commutant [3] at $\psi$ is the set

$$
C_{\psi}(D, T)=\left\{A \in B\left(L^{2}(\mathbb{R})\right): A D^{n} T^{m} \psi=D^{n} T^{m} A \psi\right\} .
$$

For each frame wavelet $\eta$, there is a unique operator $U_{\eta} \in C_{\psi}(D, T)$ such that $U_{\eta} \psi=\eta$, and $U_{\eta}^{*}$ is injective and has closed range. Moreover, $\eta$ is an orthonormal wavelet if and only if $U_{\eta}$ is unitary, while $\eta$ is a normalized tight frame wavelet if and only if $U_{\eta}^{*}$ is an isometry [5].

Two frame wavelets $\eta_{0}$ and $\eta_{1}$ are said to be uniformly path-connected if there is a path of frame wavelets $\left\{\eta_{t}: t \in[0,1]\right\}$ such that $U_{\eta_{t}}$ is a continuous path in the operator norm (and hence $\left\{\eta_{t}: t \in[0,1]\right\}$ is a continuous path in the $L^{2}$ norm). The uniform connectivity for certain classes of wavelets is related to the interpolation theory of wavelets and was investigated in several papers (cf. [3], 4]). We will prove that the path-connectedness of s-elementary frame wavelets cannot be strengthened to uniform path-connectedness. In fact, we will prove that the set of frame wavelets and the set of normalized tight frame wavelets are not uniformly path-connected either. We need the following simple lemma.

Lemma 7. Let $U$ be a unitary operator. If $V$ is an isometry such that $\|U-V\|<1$, then it must be unitary.

Proof. Write $V=U+(V-U)=U\left(I+U^{*}(V-U)\right)$. Since $\left\|U^{*}(V-U)\right\| \leq$ $\|V-U\|<1$, it follows that $\left(I+U^{*}(V-U)\right)$ is invertible. Thus $V$ is invertible and hence unitary.

Theorem 3. None of the following sets is uniformly path-connected:

(i) The set of all frame wavelets.

(ii) The set of all normalized tight frame wavelets.

(iii) The set of all s-elementary frame wavelets.

Proof. We will only prove that set (i) is not uniformly path-connected. The other two cases are similar. Let $\eta_{0}$ be a Riesz wavelet (i.e., $\left\{D^{n} T^{\ell} \eta: n . \ell \in \mathbb{Z}\right\}$ is a Riesz basis for $L^{2}(\mathbb{R})$ ), and $\eta_{1}$ a frame wavelet which is not a Riesz wavelet. We claim that $\eta_{0}$ and $\eta_{1}$ can never be uniformly path-connected. In fact, if there exist $\left\{\eta_{t}: t \in[0,1]\right\}$ such that $\left\{U_{\eta_{t}}\right\}$ is a continuous path in the operator norm, write $U(t)=U_{\eta_{t}}$ and $S(t)=U(t) U(t)^{*}$, then it is obvious that $S(t)$ is also continuous in the operator norm. Since $\eta_{t}$ is a frame wavelet, it follows that $S(t)$ (which is refereed as the frame operator in the literature; cf. [5]) is invertible for all $t$. By the continuity of the inverse operation, we have that $S(t)^{-1 / 2}$ must be continuous.

From the polar decomposition of $U(t)$, we have that $V(t)=U(t)^{*} S(t)^{-1 / 2}$ is an isometry for each $t$. Therefore $V(t)$ is a continuous path (in the operator norm) consisting of isometries. Since $\eta_{0}$ is a Riesz wavelet and $\eta$ is not, we have that $V(0)$ is unitary but $V(1)$ is an isometry which is not unitary. This implies that $V(t)$ is a continuous path connecting a unitary and a non-unitary isometry, and contradicts Lemma 7.

\section{ACKNOWLEDGEMENT}

The authors thank the referee for pointing out an error in an earlier version of the paper. 


\section{REFERENCES}

[1] X. Dai, Y. Diao and Q. Gu, Frame Wavelet Sets in $\mathbb{R}$, Proc. of Amer. Math. Soc., Vol 129 (2001), no. 7 , 2045-2055. MR 2002a:46006

[2] X. Dai, Y. Diao, Q. Gu and D. Han, Frame Wavelets in Subspaces of $L^{2}\left(\mathbb{R}^{d}\right)$, Proc. of Amer. Math. Soc., Vol 130 (2002), no. 11: 3259-3267. MR 2003f: 42052

[3] X. Dai and D. Larson, Wandering vectors for unitary systems and orthogonal wavelets, Memoirs Amer. Math. Soc., 134 (1998), no. 640. MR 98m:47067]

[4] Q. Gu, On interpolation families of wavelet sets, Proc. of Amer. Math. Soc., Vol 128 (2000), no. 10, 2973-2979. MR 2000m:42026

[5] D. Han and D. Larson, Frames, bases and group representations, Memoirs Amer. Math. Soc., 147 (2000), no. 697. MR 2001a:47013

[6] Rufeng Liang, Wavelets, their phases, multipliers and connectivity, Ph.D. Thesis, December, 1998. University of North Carolina-Charlotte.

[7] M. Paluszynski, H. Sikic, G. Weiss and S. Xiao, Tight frame wavelets, their dimension functions, MRA tight frame wavelets and connectivity properties, Adv. in Comp. Math. 18 (2003), 297-327. MR 2004b:42087

[8] D. Speegle, The s-elementary wavelets are path-connected, Proc. Amer. Math. Soc., Vol 127 (1999), no. 1, 223-233. MR 99b:42045

[9] Wutam Consortium, Basic Properties of Wavelets, Journal of Fourier Analysis and Applications, Vol 4, no. 4-5 (1998), 575-594. MR 99i:42056

Department of Mathematics, University of North Carolina at Charlotte, CharLOTTE, NorTh CAROlina 28223

E-mail address: xdai@uncc.edu

Department of Mathematics, University of North Carolina at Charlotte, CharLOTTE, North CAROlina 28223

Department of Mathematics, East China Normal University, Shanghai, People's RePUBLIC OF CHINA 200062

Department of Mathematics, University of Central Florida, Orlando, Florida 32816 